\title{
Metastatic Renal Cell Carcinoma Rapidly Progressive to Sunitinib: What to Do Next?
}

\author{
Melissa Bersanelli ${ }^{a, b, *}$, Roberto Iacovelli ${ }^{c, d}$, Sebastiano Buti ${ }^{a}$, Nadine Houede $^{e}$, \\ Brigitte Laguerre $^{f}$, Giuseppe Procopio ${ }^{g}$, Stéphanie Lheureux ${ }^{h}$, R. Fischer ${ }^{i}$, Sylvie Negrier ${ }^{j}$, \\ Alain Ravaud ${ }^{k}$, Stéphane Oudard ${ }^{l}$, Bernard Escudier ${ }^{c}$, Laurence Albiges $^{c}$, Camillo Porta $^{m}$ \\ ${ }^{a}$ Medical Oncology Unit, University Hospital of Parma, Parma, Italy; ${ }^{\mathrm{b}}$ Department of Medicine and Surgery, University of Parma, Parma, Italy; ${ }^{\mathrm{c}}$ Medical Oncology \\ Department, Institut Gustave Roussy, Villejuif, France; ${ }^{\mathrm{d}}$ Oncology Unit B, Sapienza University of Rome, Rome, Italy; ${ }^{\mathrm{e}}$ Medical Oncology Department, Institut \\ Bergonie, Bordeaux, France; ${ }^{\mathrm{f}}$ Medical Oncology Department, Centre Eugène Marquis, Rennes, France; ${ }^{\mathrm{g}}$ IRCCS Istituto Nazionale Tumori, Milano, Italy; ${ }^{\mathrm{h}}$ Medical \\ Oncology Department, Centre François Baclesse, Caen, France; ${ }^{i}$ Medical Oncology Department, The Royal Marsden Hospital, London, UK; ${ }^{j}$ Medical Oncology \\ Department, University of Lyon, Centre Léon Bérard, Lyon, France; ${ }^{\mathrm{k}}$ Medical Oncology Department, Bordeaux University Hospital, Saint-André Hospital, Bordeaux, \\ France; ${ }^{1}$ Medical Oncology Department, Hopital Europeen Georges Pompidou, Paris, France; ${ }^{\mathrm{m}}$ Medical Oncology, IRCCS Policlinico San Matteo, Pavia, Italy
}

\section{Article info}

\section{Article history:}

Accepted June 19, 2019

Associate Editor:

Paul Nguyen

\section{Keywords:}

Primary refractory

Primary resistance

Rapidly progressive

Renal cell carcinoma

Sunitinib

Tyrosine kinase inhibitors

\begin{abstract}
Background: From $10 \%$ to $26 \%$ of patients with metastatic renal cell carcinoma (mRCC) experience rapidly progressive disease (PD) on treatment with sunitinib.

Objective: To investigate the benefit of subsequent treatment with another tyrosine kinase inhibitor (TKI) or a mammalian target of rapamycin (mTOR) inhibitor in such primary refractory patients.

Design, setting, and participants: A total of $150 \mathrm{mRCC}$ patients with rapidly PD on firstline sunitinib (within two cycles, $n=93$, or four cycles, $n=57$ ) were identified: median age $59 \mathrm{yr}$; nephrectomy 86\%; histological subtypes: clear cell (77.8\%), papillary (14\%), and sarcomatoid features (18\%); according to the Memorial Sloan-Kettering Cancer Center and French classifications: good risk (11\% and 7\%, respectively), intermediate (68\% and 63\%, respectively), and poor ( $21 \%$ and $29 \%$, respectively).

Outcome measurements and statistical analysis: Data were retrospectively collected by a questionnaire from 19 European oncology centers between March 2005 and March 2011. Progression-free survival (PFS) and overall survival (OS) were calculated (Kaplan-Meier method). Results and limitations: Median OS from the start of first-line treatment was 7.4 mo. Second-line treatment was administered to 86 (57\%) patients (44 mTOR inhibitors: 23 everolimus and 21 temsirolimus; 39 TKIs alone or in combination; three chemotherapy). Secondline PFS was not significantly different between TKIs and mTOR inhibitors (2.0 vs $0.9 \mathrm{mo}$; $p=0.536$ ). Median OS from the start of second-line treatment was 5.0 mo for mTOR inhibitors and 6.6 mo for TKIs $(p=0.15)$.

Conclusions: Treatment with further TKIs or mTOR inhibitors for mRCC patients primarily refractory to first-line sunitinib in the observed time period achieved very minimal benefit, suggesting avoiding TKI rechallenge and possibly preferring alternative strategies, such as immune checkpoint inhibitors, after PD to a treatment line including a TKI in this setting.

Patient summary: The present work collected data about 150 patients affected by metastatic renal cell carcinoma, who received one of the current standard of care as first-line treatment, namely, the antiangiogenic drug sunitinib, and experienced rapid worsening of the disease. We investigated and described the subsequent outcome of such patients treated with two different types of drug, administered as second-line therapy, to better understand the best strategy to adopt for patients who got no benefit from sunitinib and to describe the current therapeutic approach in such cases.

(C) 2019 European Association of Urology. Published by Elsevier B.V. All rights reserved.

* Corresponding author. Medical Oncology, University Hospital of Parma, Via Gramsci 14, Parma 43126, Italy. Tel. +39 0521 702316, +39 331 3581097; Fax: +39 0521702660. E-mail address: bersamel@libero.it (M. Bersanelli).
\end{abstract}

https://doi.org/10.1016/j.euo.2019.06.018

2588-9311/@ 2019 European Association of Urology. Published by Elsevier B.V. All rights reserved. 


\section{Introduction}

Targeted therapy with tyrosine kinase inhibitors (TKIs) has increased overall survival (OS) of patients with metastatic renal carcinoma (mRCC) [1,2]. However, a subgroup of patients treated with such agents never experiences tumor shrinkage, and can be identified as harboring a "primary refractory disease" or a rapidly progressive disease (PD) to TKI therapy [3]. Despite the current availability of new treatment options, such as immune checkpoint inhibitors (CKIs), the sequential use of oral drugs and the rechallenge of further TKIs are still currently indispensable for patients with a longer course of disease, while no data have yet been provided for CKI rechallenge. In this light, it would be useful to understand which type of subsequent mechanism of action (MOA) could be exploited for primary TKI-refractory patients.

Primary refractory disease has no standard definition, and this may partly account for the few studies available on patients with rapidly PD [3-5]. The terms are both commonly used to refer to patients in whom the best response is disease progression according to the Response Criteria in Solid Tumors (RECIST). Two series have been reported to date focusing on this population: Heng et al. [4] identified 272 primary refractory cases among a cohort of 1056 patients receiving TKI (incidence rate, 26\%); Busch et al. [5] reported 35 cases with intrinsic resistance to TKIs among a cohort of 189 patients (18.5\%).

In large randomized studies reported with vascularendothelial growth factor receptor (VEGFR) inhibition as first-line treatment, the incidence of PD as the best response was $10.3-12 \%$ for sorafenib [6,7], $18 \%$ for pazopanib [8], $20 \%$ for bevacizumab plus interferon- $\alpha$ [9], $21 \%$ for sunitinib [2], $13.1 \%$ for tivozanib [10], and $18 \%$ for cabozantinib [11].

Owing to of the feeling that primary resistance to sunitinib will apply to other TKIs, the most common treatment strategy for patients with rapidly PD in the pre-CKI era was the use of mammalian target of rapamycin (mTOR) inhibitors, despite the lack of evidence for the efficacy of such switch in this setting. Given the possibility to choose the CKI nivolumab as next-line therapy, the issue would now be simple, if it were not for the current availability of a new multitarget TKI, namely, cabozantinib, which gives the chance to overcome the prior TKI resistance (through MET and AXL inhibition), demonstrating lower rates of primary refractory cases when indirectly compared with nivolumab as second-line therapy (12\% vs $35 \%$ ) [12-14].

Considering the currently ongoing shift of CKIs in the firstline setting of mRCC treatment $[15,16]$ and, on the other hand, the possible applicability of cabozantinib as first-line option in certain subgroups (poor/intermediate-risk patients) [11], what to do in second or subsequent treatment lines after a prior TKI still remains an actuarial issue.

The aim of the present study was therefore to analyze the outcome of second-line treatment of patients rapidly progressing (within $24 \mathrm{wk}$ ) on first-line sunitinib therapy, representing the gold standard for mRCC primary treatment at the time of the study planning, to determine whether the TKI rechallenge strategy, or the mTOR-inhibition switch, still has any clinical rationale.

\section{Patients and methods}

\subsection{Patients}

We performed a retrospective analysis of data on patients with mRCC who experienced rapidly PD on first-line sunitinib treatment, defined as PD within $24 \mathrm{wk}$ (four cycles) from starting therapy and without tumor shrinkage after two cycles of sunitinib. Data were collected by sending a questionnaire to contact oncologists from the French Kidney Cancer Group, Italian Nephro-Oncology Group, and Royal Marsden Hospital (UK) for distribution to their members. Members had to complete the questionnaire for all patients with mRCC fulfilling all the following criteria: (1) patients with histologically proven mRCC; (2) patients who had been treated with first-line sunitinib between March 2005 and March 2011, according to standard schedule (4 wk on, $2 \mathrm{wk}$ off cycle); (3) patients who had progressed within $24 \mathrm{wk}$ (four cycles) from starting therapy and without tumor shrinkage after two cycles of sunitinib; and (4) patients with documented baseline characteristics and adequate follow-up. In all cases, informed consent must have been obtained for therapy administration.

Data regarding patients and their tumors were retrospectively collected. Patients were classified according to the Memorial Sloan-Kettering Cancer Center (MSKCC) modified criteria [17] and also according to the French prognostic criteria [18,19] (performance status [PS] $>0$, number of metastatic sites $>1$, interval between initial diagnosis of mRCC and systemic treatment $<1 \mathrm{yr}$, and presence of liver metastases). The principles outlined in the Declaration of Helsinki have been followed for the present work.

\subsection{Statistical analysis}

OS was calculated from the start of sunitinib to death or the last followup. For patients who received a second-line treatment, second-line survival was also calculated from the start of second line to death or the last follow-up. In addition, progression-free survival (PFS) for second line was defined as the interval from the start of second-line therapy to the first documentation of disease progression or death from any cause, whichever occurred first.

All values were examined as binary variables. Multivariate analysis was performed using the Cox proportional hazard model, and a stepwise selection algorithm that used a type I error of 0.05 for model entry and 0.10 for elimination. Additional elimination was applied to identify significant variables at the level of $p<0.05$. The chi-square test was used to assess the differences. We used Predictive Analytics SoftWare (PASW, v 18; IBM SPSS).

\section{Results}

Overall, 150 patients (mean age, 58 yr [range 22-83]) from 19 major European oncology centers were included in this analysis. Their baseline characteristics are presented in Table 1 . The mean time from surgery to the diagnosis of metastatic disease was $11 \mathrm{mo}$, including 78 patients $(52 \%)$ with synchronous metastatic disease at diagnosis.

\subsection{Response to first-line treatment with sunitinib}

All 150 patients received sunitinib as first-line therapy; 133 (89\%) received a standard dose of $50 \mathrm{mg} 4 \mathrm{wk}$ on followed by 2 wk off. Eleven percent of patients started at a lower dose of $37.5(n=13,8.7 \%)$ or $25 \mathrm{mg} / \mathrm{d}(n=4 ; 2.7 \%)$.

Two-thirds of the patients $(n=93,62.2 \%)$ stopped treatment within the first two cycles of treatment for PD (12 wk), and the remainder $(n=57,37.8 \%)$ received up to 


\section{Table 1 - Patient characteristics}

\begin{tabular}{|c|c|}
\hline Characteristics & Patients \\
\hline Male/female & $115 / 35$ \\
\hline Age (yr) & $58(22-83)$ \\
\hline \multicolumn{2}{|l|}{ Disease characteristics (\%) } \\
\hline $\mathrm{T} 1$ & 6 \\
\hline $\mathrm{T} 2$ & 14 \\
\hline T3 & 58 \\
\hline $\mathrm{T} 4$ & 8.7 \\
\hline NA & 13.3 \\
\hline NO & 26.7 \\
\hline N1 & 10.7 \\
\hline $\mathrm{N} 2$ & 21.3 \\
\hline NA & 41.3 \\
\hline \multicolumn{2}{|l|}{ Histology (\%) } \\
\hline Clear cell & 77 \\
\hline Papillary & 13.5 \\
\hline Pure sarcomatoid & 5.4 \\
\hline Sarcomatoid component & 13 \\
\hline Others & 4 \\
\hline Metastatic at diagnosis (\%) & 49 \\
\hline \multicolumn{2}{|l|}{ Number of metastatic sites (\%) } \\
\hline 1 & 19 \\
\hline 2 & 33 \\
\hline$\geq 3$ & 48 \\
\hline \multicolumn{2}{|l|}{ Sites of metastasis (\%) } \\
\hline Lung & 70 \\
\hline Lymph node & 59 \\
\hline Bone & 31 \\
\hline Liver & 25 \\
\hline Brain & 11 \\
\hline Renal bed & 9 \\
\hline \multicolumn{2}{|l|}{ MSKCC classification (\%) } \\
\hline Good & 10.7 \\
\hline Intermediate & 63.7 \\
\hline Poor & 18.9 \\
\hline NA & 6.7 \\
\hline \multicolumn{2}{|l|}{ French classification (\%) } \\
\hline Good & 7.7 \\
\hline Intermediate & 62 \\
\hline Poor & 28.3 \\
\hline NA & 2 \\
\hline
\end{tabular}

four cycles (Fig. 1). Assessment of these 57 patients after two cycles identified 29 patients with PD who received two further cycles despite PD on evaluation and 28 with stable disease (SD) according to the RECIST criteria but without any tumor shrinkage, consistent with the inclusion criteria. Median OS in the overall population was 7.4 mo (6.6-8.2; Fig. 2). OS was significantly shorter in the group that received two cycles than in the group receiving four cycles (6.0 [5.2-6.8] vs 9.3 [7.4-11.3] mo; $p=0.008)$.

\subsection{Response to second-line treatments}

Of the 150 patients, 86 (57.3\%) received second-line treatment. The reasons for no treatment for the remaining $64(42.7 \%)$ were a poor PS related to disease progression $(n=55)$, diagnosis of brain metastases $(n=3)$, surgery $(n=1)$, patient refusal $(n=1)$, unaffordable treatment $(n=1)$, and unknown $(n=3)$. Second-line response rates were $0 \%$ for complete response, $10 \%$ for partial response, and $27 \%$ for SD. Only 22 patients (25.5\%) experienced a clinical benefit lasting for $>3 \mathrm{mo}$.
At the time of analysis, 23/150 patients (15.3\%) were alive. Median second-line PFS and second-line survival were 1.6 (0.5-2.7) and 5.9 (4.3-7.4) mo, respectively (Fig. 3). Median OS was significantly longer in patients who received second-line treatment than in those who did not (10.5 [8.0$13.1]$ vs 4.1 [2.8-5.5] mo; log-rank test, $p<0.0001$ ).

Among the 86 patients receiving second-line treatment, 44 received an mTOR inhibitor (21 everolimus and 23 temsirolimus), 39 received a TKI either alone or in combination (35 sorafenib, two axitinib, and two sunitinib plus bevacizumab), and three patients received chemotherapy. The groups receiving mTOR and TKI as second-line targeted therapy had similar baseline characteristics (Table 2). Median second-line survival (6.6 vs $5.0 \mathrm{mo}$; $p=0.157$ ) and second-line PFS (2.0 vs 0.9 mo; log-rank test, $p=0.536)$ were not significantly different between TKI and mTOR inhibitors (Table 3 and Fig. 4).

\subsection{Prognostic variables}

Variables, including Karnofsky performance status (KPS) of $<80$, absence of nephrectomy, increased baseline value for lactate dehydrogenase (LDH), MSKCC poor-risk group, number of metastatic sites $>1$, absence of second-line treatment, and number of cycles ( 2 vs 4 cycles), were associated with worse OS in univariate analyses. In a multivariate analysis, a KPS of $<80$, high baseline values for $\mathrm{LDH}$, and corrected calcium were found to be independent prognostic factors.

\subsection{Exploratory analysis}

Among the 150 patients, 122 exhibited PD at two-cycle computed tomography scan, while 28 were nonprogressive patients with no tumor shrinkage according to the inclusion criteria (Fig. 5A). Patients who had PD at two-cycle evaluation had median OS of $6.6 \mathrm{mo}$, while patients with SD at two cycles had median OS of $9.3 \mathrm{mo}$ ( $p=0.12$; Fig. 5B). Among the 122 patients with PD at cycle 2, 93 stopped sunitinib with median OS of 5.9 mo and 29 patients who received two further cycles of sunitinib had 10.0-mo OS $(p=0.043$; Fig. $5 \mathrm{C})$. It is noteworthy that patients who received a second-line treatment after discontinuation due to progression had OS of 5.2 versus 5.1 mo for patients who stayed on sunitinib despite PD at two cycles $(p=0.9$; Fig. 5D).

To investigate the impact of PD timing, the population of patients who received four cycles of sunitinib but experienced PD with two cycles $(n=29)$ was compared with those who experienced PD within four cycles while non-PD after two cycles $(n=28)$. There was no difference in OS between these two populations ( 9.3 vs $10 \mathrm{mo} ; p=0.9$ ).

\section{Discussion}

Despite recent advances in the treatment of patients with mRCC, patients with rapidly PD on TKI treatment represent a relatively substantial subset of patients with a very poor prognosis. The rate of primary resistance, defined as PD as 


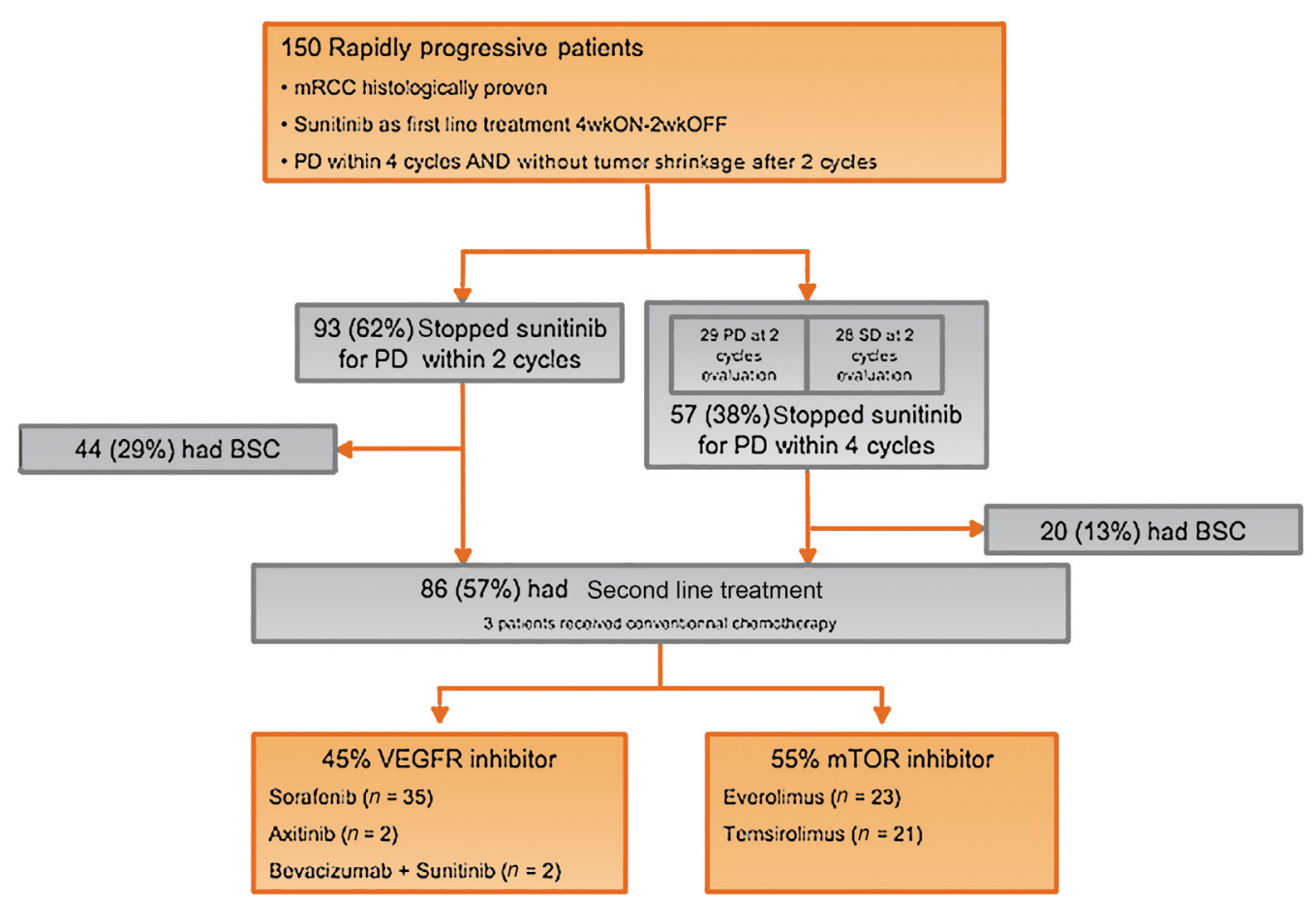

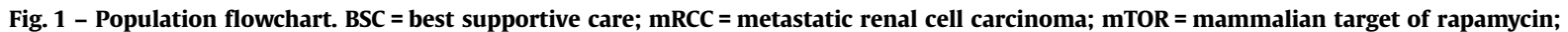
$\mathrm{PD}=$ progressive disease; $\mathrm{SD}=$ stable disease; VEGFR = vascular-endothelial growth factor receptor; $4 \mathrm{wkON}-2 \mathrm{wkOFF}=4 \mathrm{wk}$ on, 2 wk off cycle.

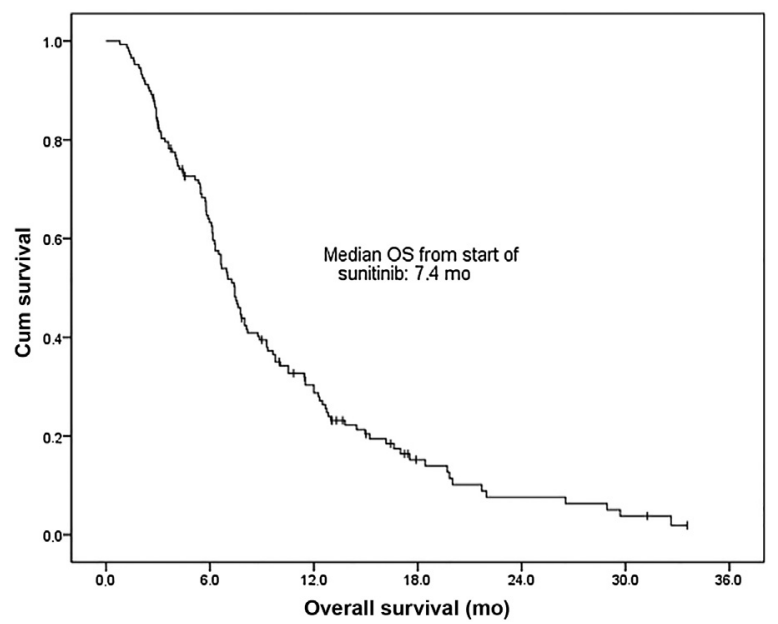

Fig. 2 - Overall survival. Cum = cumulative; $O S=$ overall survival.

the best response, was $21 \%$ in the pivotal phase III trial of sunitinib [2], and it was confirmed in the two retrospective studies focusing on this subpopulation by Heng et al. [4] and Busch et al. [5] (rates of $26 \%$ and $18.5 \%$, respectively). Since in clinical practice about one patient out of five do not benefit from treatment, the issue of the prognosis and decision of further treatment must be enlightened.

In the present study, which used $24 \mathrm{wk}$ as a time limit to define refractory disease, median OS of the entire cohort of 150 patients was 7.4 mo. These data are consistent with those from Heng et al.'s [4] cohort (6.8 mo). Regarding second-line treatment options, Heng et al. [4] reported a $40 \%$ rate of second-line treatment, while we report a $57 \%$ rate. In our experience, there was no difference in secondline PFS or OS regardless of the choice of a TKI or an MTOR inhibitor, in line with Heng et al.'s [4] findings. It must be underlined that we provide the most homogeneous comparison between the type of second-line treatments regarding the number of patients (39 vs 44) as well as prognosis characteristics (Table 4 ).

In our cohort, the overall benefit of a second-line treatment, regardless of its nature, is highly questionable with second-line PFS of $1.6 \mathrm{mo}$ and second-line survival of 5.9 mo, consistent with those previously reported [4].

Furthermore, it is still not possible to predictively identify patients refractory to sunitinib. In our population, the only prognostic element isolated in multivariate analysis was the MSKCC score, with the limitations due to the retrospective design of this study using data from questionnaires.

Of note, the selection of progressive patients within four cycles of treatment provides us with information on three more aspects regarding this subpopulation:

The comparison among patients with PD at two cycles between those who benefit from an alternative second line $(n=49)$ and those who stayed on sunitinib for two more cycles despite progression $(n=29)$ stated no statistical difference in favor of a therapeutic change to an alternative second line (Fig. 5D). It is therefore highly questionable whether switching to another drug rather than staying on sunitinib despite PD is useful. Nevertheless, this finding could be partially related to a selection bias in favor of patients staying on sunitinib therapy, probably identified by the clinicians as individuals with a clinical benefit despite PD. 

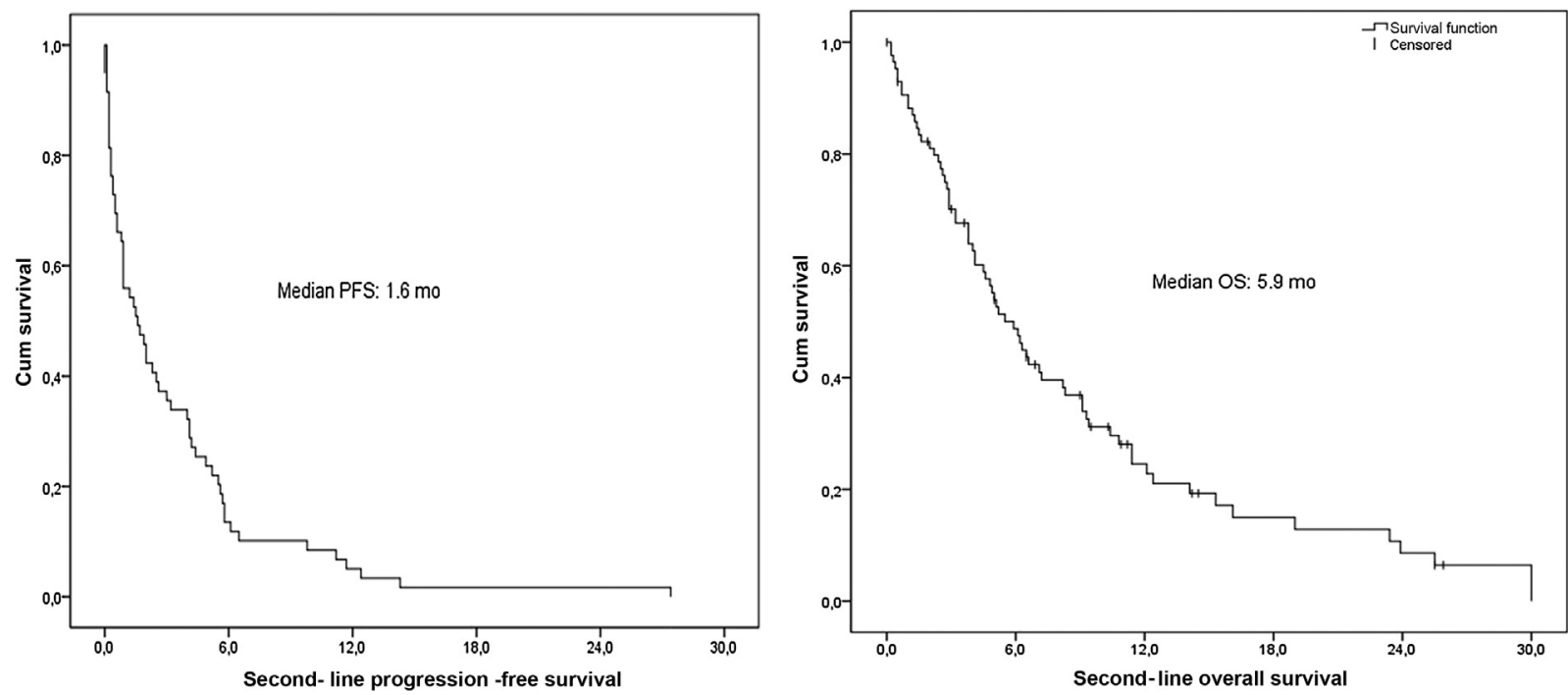

Fig. 3 - Second-line PFS and OS (calculated from the start of second line). Cum = cumulative; PFS = progression-free survival; oS = overall survival.

Table 2 - Comparison between best supportive care (BSC) and second-line treatment

\begin{tabular}{|c|c|c|c|}
\hline Characteristics & $\begin{array}{c}\text { BSC } \\
(n=64)\end{array}$ & $\begin{array}{l}\text { 2nd line } \\
(n=86)\end{array}$ & $\begin{array}{c}\text { Chi-square test } \\
\text { ( } p \text { value })\end{array}$ \\
\hline Males (\%) & 73.4 & 67.4 & 0.4 \\
\hline Age (yr) & $59(28-83)$ & $57(23-76)$ & 0.4 \\
\hline \multicolumn{4}{|l|}{ Disease characteristics } \\
\hline Histology (\%) & & & 0.6 \\
\hline Cellules clear & 79.0 & 75.6 & \\
\hline Other histologies & 21.0 & 23.4 & \\
\hline Sarcomatoid features (\%) & 16.1 & 19.8 & 0.6 \\
\hline Number of metastatic sites (\%) & & & 0.3 \\
\hline 1 & 21.9 & 17.4 & \\
\hline 2 & 29.7 & 36.0 & \\
\hline$\geq 3$ & 48.4 & 46.6 & \\
\hline \multicolumn{4}{|l|}{ Sites of metastasis (\%) } \\
\hline Lung & 75.0 & 66.3 & 0.2 \\
\hline Abdominal nodes & 25.0 & 47.7 & 0.005 \\
\hline Liver & 26.6 & 24.4 & 0.8 \\
\hline Bone & 31.3 & 31.4 & 0.9 \\
\hline \multicolumn{4}{|l|}{ Prognostic factors (\%) } \\
\hline Interval diagnosis-sunitinib $<1 \mathrm{yr}$ & 67.2 & 77.9 & 0.1 \\
\hline Karnofsky PS $<80 \%$ & 26.9 & 7.1 & 0.001 \\
\hline Ca correct $>$ UNR & 11.9 & 4.8 & 0.1 \\
\hline Hemoglobin $<$ LNR & 61.9 & 38.1 & 0.004 \\
\hline LDH 1.5 UNR & 21.1 & 13.4 & 0.2 \\
\hline MSKCC classification (\%) & & & 0.08 \\
\hline Good & 10.2 & 12.2 & \\
\hline Intermediate & 59.3 & 74.4 & \\
\hline Poor & 30.5 & 13.4 & \\
\hline French classification (\%) & & & 0.4 \\
\hline Good & 7.9 & 7.1 & \\
\hline Intermediate & 55.6 & 69.1 & \\
\hline Poor & 36.5 & 23.8 & \\
\hline
\end{tabular}

Continuing treatment for two extra cycles when PD is diagnosed at first evaluation, we identified that patients receiving four cycles of sunitinib despite a lack of response after two cycles $(n=29)$ had better OS than those who experienced PD and stopped sunitinib $(n=93 ; 10.1$ vs $5.9 \mathrm{mo}, p=0.049$ ). This comparison is allowed as the percentage of patients who were referred to best supportive care $(47.8 \%$ vs $41.8 \%)$ is similar in both groups (Fig. 5 C).
Therefore, we hypothesized that the difference in OS may indeed be related to the longer exposure to sunitinib.

The trend in OS between patients who exhibit PD within two cycles $(n=122)$ versus those who exhibited SD at first evaluation (with no tumor shrinkage; 6.6 vs $9.3 \mathrm{mo}$ ) was not significant $(p=0.12$; Fig. 5B). About the meaning of developing PD after two or four cycles, there was no difference in OS between patients developing PD at these 
Table 3 - Second-line progression-free survival (PFS) and second-line survival (OS)

\begin{tabular}{|c|c|c|c|c|c|c|}
\hline \multirow[t]{2}{*}{ Class of second line } & \multirow[t]{2}{*}{$n$} & \multicolumn{2}{|c|}{ 2nd-line survival } & \multirow[t]{2}{*}{$n$} & \multicolumn{2}{|c|}{ 2nd-line PFS } \\
\hline & & Value $^{\mathrm{a}}$ & $95 \% \mathrm{CI}$ & & Value $^{a}$ & $95 \% \mathrm{Cl}$ \\
\hline TKI & 39 & 6.6 & $4.8-8.5$ & 22 & 2.0 & $0.3-3.6$ \\
\hline mTOR & 44 & 5.0 & $3.0-7.0$ & 36 & 0.9 & $0.1-1.9$ \\
\hline Global & 83 & 5.9 & $4.4-7.4$ & 58 & 1.6 & $0.3-2.9$ \\
\hline
\end{tabular}
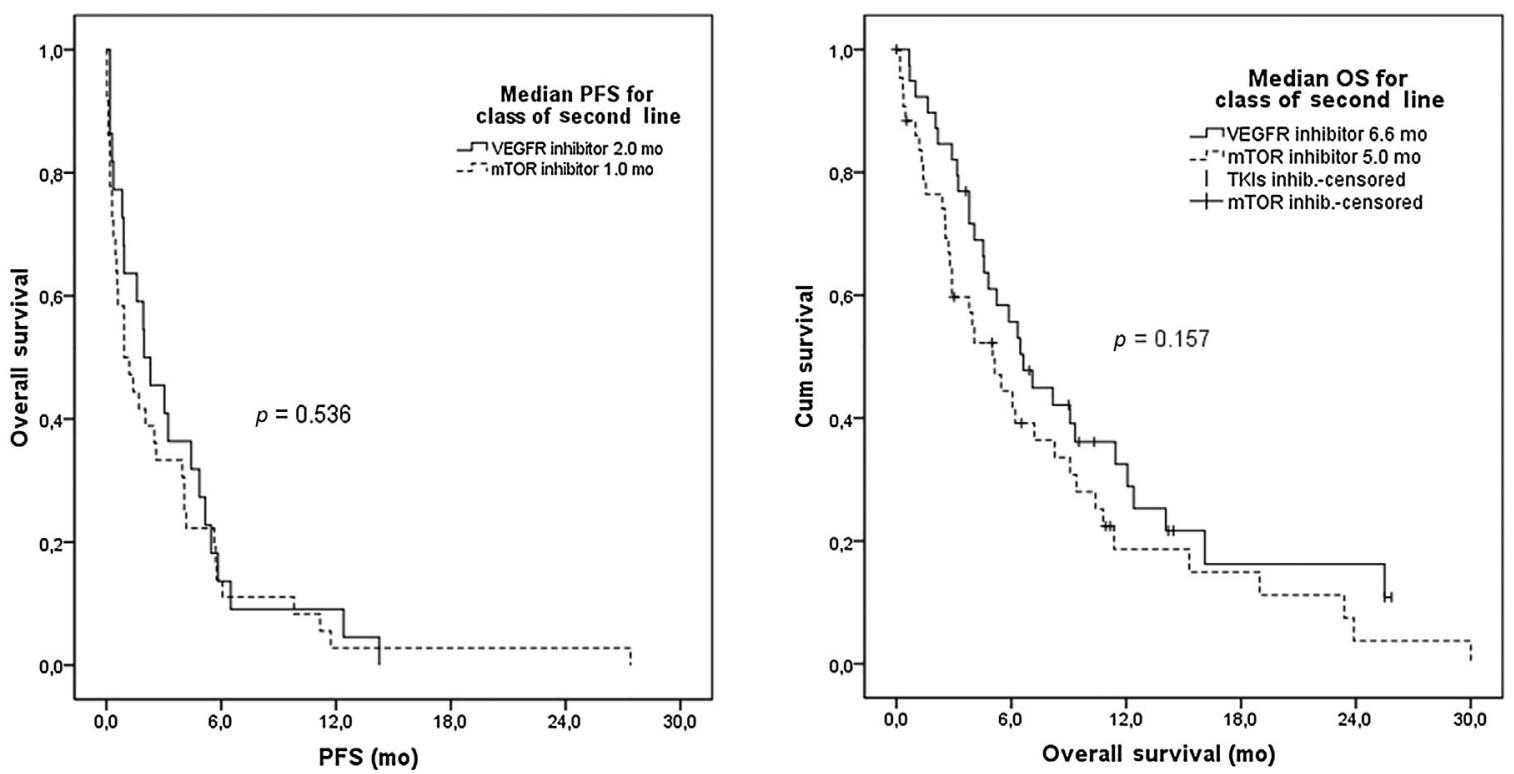

Fig. 4 - Second-line PFS and second-line OS according to the nature of treatment. Cum = cumulative; inhib. = inhibitor; $\mathbf{m T O R}=$ mammalian target of rapamycin; PFS = progression-free survival; OS = overall survival; TKI = tyrosine kinase inhibitor; VEGFR = vascular-endothelial growth factor receptor.

A

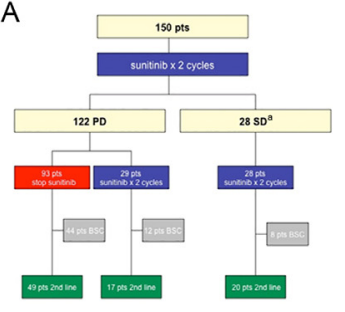

B

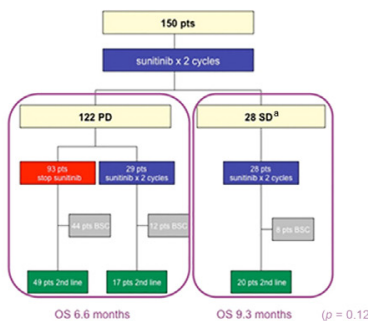

C

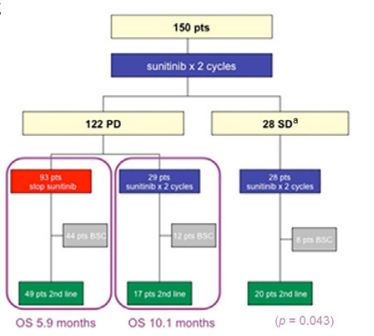

D

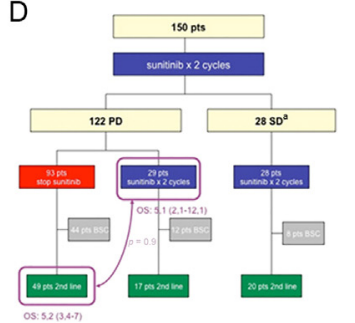

Fig. 5 - Exploratory analysis. (A) Overall population. (B) Prognosis of patients with SD versus PD within two first cycles of sunitinib a SD with no tumor shrinkage. (C) Prognosis of patients with PD at two cycles: difference between sunitinib arrest and sunitinib continuation. (D) Patients with PD at two cycles: similar prognosis of patients with second-line treatment versus sunitinib continuation. $\mathrm{BSC}=$ best supportive care; OS = overall survival; $\mathrm{PD}=$ progressive disease; $\mathrm{pts}=$ patients; $\mathrm{SD}=$ stable disease. ${ }^{\mathrm{a}} \mathrm{SD}$ with no tumor shrinkage.

two different time points of a four-cycle treatment (9.3 vs $10 \mathrm{mo} ; p=0.9$ ), raising the hypothesis that whether the PD was occurring earlier or later within the first 6 mo did not impact OS. Furthermore, this observation is in favor of defining primary resistance not only in PD occurring within 3 mo but also extending the definition to the first 6 mo of sunitinib treatment.

The first two highlighted points did not collide with the evidence that the currently available alternative TKI cabozantinib can achieve a benefit irrespective of primary resistance to first-line TKI: in fact, it is uncertain whether the inhibition of MET, RET, or AXL drives the major clinical activity of cabozantinib or whether the benefit is simply due to a VEGFR inhibitory effect [20]. Interestingly, cabozantinib efficacy seems to be independent of MET expression and by its typical "VEGF inhibition-related" toxicity profile. These previous data, together with our current findings, suggest that maintaining "VEGF pressure" can still slow disease progression irrespective of the primary sensitivity to TKI [21]. 
Table 4 - Second-line characteristics between mTOR group and VEGFR inhibitor group

\begin{tabular}{llll}
\hline Characteristics & $\begin{array}{c}\text { VEGFR inhibitor } \\
(n=39)\end{array}$ & $\begin{array}{c}\text { mTOR inhibitor } \\
(n=44)\end{array}$ & $\begin{array}{c}\text { Chi-square test } \\
(p \text { value })\end{array}$ \\
\hline Males (\%) & 89.7 & 70.5 & 0.03 \\
Age (yr) & $57(31-83)$ & $56(28-78)$ & 0.5 \\
Histology (\%) & 76.9 & 75.0 & 0.8 \\
$\quad$ Cellules clear & 23.1 & 25.0 & 0.5 \\
$\quad$ Other histologies & 15.4 & 20.5 & 0.9 \\
Sarcomatoid features (\%) & & 56.8 & \\
Number of sunitinib cycles (\%) & 54.4 & 43.2 & \\
2 & 43.6 & \\
4 & & \\
\hline mTOR = mammalian target of rapamycin; VEGFR = vascular-endothelial growth factor receptor. & \\
\hline
\end{tabular}

Finally, we provided informative observations on pathological characterization: our population included a relatively high proportion of tumors with sarcomatoid features (18\%) compared with historical cohorts [22,23], underlining the interest for different therapeutic strategies in these poorprognosis subtypes, which may benefit from conventional chemotherapy [24] and possibly from the use of CKIs [25].

The design of our study has several caveats. First, the study cohort is outdated, referring to years 2005-2011 and lacking cases treated with second-line nivolumab or cabozantinib; thus, it is limited to providing data about classical therapeutic options (such as "old" TKIs and mTOR inhibitors), beyond the obvious possibility to treat TKIrefractory patients with the new immunotherapy, in case they have not received it previously. Then, the study was retrospective, relied on the analysis of data from questionnaires, and the number of patients did not exceed 150 , although the patients were from many oncology centers in three countries. Our study nevertheless has several strengths. We used a reproducible definition for primary refractory disease (progression within four cycles of treatment); the numbers of patients receiving TKIs or mTOR inhibitors as second-line treatment were well balanced; we applied two different classifications for prognosis; details were available on tumor histology.

\section{Conclusions}

Rapidly progressive mRCC patients can be considered having a similar dismal prognosis to poor-prognosis patients. The TKI or mTOR switch strategy might not be superior to continued treatment with sunitinib in this setting. Nevertheless, given the current rapidly changing treatment landscape for first-line approach in mRCC, the setting represented in this study is going to be outdated. Moreover, the present study only reported data about sunitinib, with no clear applicability for pazopanib, and does not provide data about the new multitarget TKI cabozantinib. Actually, our findings can still be useful to suggest only a change of the MOA, considering immunotherapy as possibly the best management option for primary refractory patients to TKI, in view of their poor prognosis and the lack of efficacy of "old" second-line treatments.
Author contributions: Melissa Bersanelli had full access to all the data in the study and takes responsibility for the integrity of the data and the accuracy of the data analysis.

Study concept and design: Albiges, Iacovelli, Porta, Escudier.

Acquisition of data: Albiges, Iacovelli, Porta, Houede, Laguerre, Procopio, Lheureux, Fischer, Negrier, Ravaud, Oudard, Escudier.

Analysis and interpretation of data: Albiges, Iacovelli, Porta, Houede, Laguerre, Procopio, Lheureux, Fischer, Negrier, Ravaud, Bersanelli, Buti, Oudard, Escudier.

Drafting of the manuscript: Albiges, Porta, Bersanelli, Buti, Escudier.

Critical revision of the manuscript for important intellectual content: Albiges, Iacovelli, Porta, Houede, Laguerre, Procopio, Lheureux, Fischer, Negrier, Ravaud, Bersanelli, Buti, Oudard, Escudier.

Statistical analysis: Iacovelli, Houede, Laguerre, Lheureux, Fischer.

Obtaining funding: None.

Administrative, technical, or material support: None.

Supervision: Albiges, Porta, Escudier.

Other: None.

Financial disclosures: Melissa Bersanelli certifies that all conflicts of interest, including specific financial interests and relationships and affiliations relevant to the subject matter or materials discussed in the manuscript (eg, employment/affiliation, grants or funding, consultancies, honoraria, stock ownership or options, expert testimony, royalties, or patents filed, received, or pending), are the following: L. Albiges and R. Iacovelli: none. C. Porta stated to have received research grants from Bayer Schering Pharma and Novartis Pharma, as well as honoraria from Bayer Schering Pharma, Pfizer Oncology, Hoffman La Roche, GSK and Novartis Pharma. N. Houede, B. Laguerre, G. Procopio, S. Lheureux, and R. Fischer: none. S. Negrier stated honorarium from Pfizer, GSK, Roche, and Novartis. A. Ravaud stated to be a member of global European and French advisory boards on urological tumors and medical treatments for Novartis, Pfizer, Bayer Schering, GSK, Roche, and Dendreon; and received institutional grants from Novartis and Roche. S. Buti received honoraria as a speaker at scientific events and for advisory role by Brystol-Myers Squibb (BMS), Pfizer, MSD, Ipsen, Roche, Eli-Lilly, AstraZeneca, and Novartis; and also received research funding from Novartis. M. Bersanelli received honoraria as a speaker at scientific events by Brystol-Myers Squibb (BMS) and Pfizer; as a consultant for advisory role by Novartis, Mundipharma, BMS and Pfizer; as a medical writer by Mundipharma; and also received research funding from Seqirus, Pfizer, Novartis, and BMS, and travel expenses for scientific events from BMS, Pfizer, MSD, Ipsen, Roche, Eli-Lilly, and Novartis. S. Oudard: none. B. Escudier stated honoraria from Bayer, Roche, Pfizer, Wyeth, and Novartis.

Funding/Support and role of the sponsor: None. 


\section{References}

[1] Rini BI, Campbell SC, Escudier B. Renal cell carcinoma. Lancet 2009;373:1119-32.

[2] Motzer RJ, Hutson TE, Tomczak P, et al. Sunitinib versus interferon alfa in metastatic renal-cell carcinoma. N Engl J Med 2007;356: $115-24$.

[3] Rini BI, Atkins MB. Resistance to targeted therapy in renal-cell carcinoma. Lancet Oncol 2009;10:992-1000.

[4] Heng DY, Mackenzie MJ, Vaishampayan UN. Primary anti-vascular endothelial growth factor (VEGF)-refractory metastatic renal cell carcinoma: clinical characteristics, risk factors, and subsequent therapy. Ann Oncol 2012;23:1549-55.

[5] Busch J, Seidel C, Weikert S, et al. Intrinsic resistance to tyrosine kinase inhibitors is associated with poor clinical outcome in metastatic renal cell carcinoma. BMC Cancer 2011;11:295

[6] Escudier B, Szczylik C, Hutson TE, et al. Randomized phase II trial of first-line treatment with sorafenib versus interferon Alfa-2a in patients with metastatic renal cell carcinoma. J Clin Oncol 2007;27:1280-9.

[7] Escudier B, Eisen T, Stadler WM, et al. Sorafenib in advanced clearcell renal-cell carcinoma. N Engl J Med 2007;356:125-34.

[8] Sternberg CN, Davis ID, Mardiak J, et al. Pazopanib in locally advanced or metastatic renal cell carcinoma: results of a randomized phase III trial. J Clin Oncol 2010;28:1061-8.

[9] Escudier B, Pluzanska A, Koralewski P, et al. Bevacizumab plus interferon alfa-2a for treatment of metastatic renal cell carcinoma: a randomised, double-blind phase III trial. Lancet 2007;370:2103-11.

[10] Motzer RJ, Nosov D, Eisen T, et al. Tivozanib versus sorafenib as initial targeted therapy for patients with metastatic renal cell carcinoma: results from a phase III trial. J Clin Oncol 2013;31:3791-9.

[11] Choueiri TK, Halabi S, Sanford BL, et al. Cabozantinib versus sunitinib as initial targeted therapy for patients with metastatic renal cell carcinoma of poor or intermediate risk: the Alliance A031203 CABOSUN trial. J Clin Oncol 2017;35:591-7.

[12] Grassi P, Verzoni E, Mennitto A, Procopio G. Cabozantinib in advanced renal cell carcinoma: a METEOR impact on clinical practice. Transl Androl Urol 2016;5:974-6.

[13] Choueiri TK, Escudier B, Powles T, et al. Cabozantinib versus everolimus in advanced renal-cell carcinoma. N Engl J Med 2015;373:1814-23.
[14] Motzer RJ, Escudier B, McDermott DF, et al. Nivolumab versus everolimus in advanced renal-cell carcinoma. $N$ Engl J Med 2015;373:1803-13.

[15] Motzer RJ, Tannir NM, McDermott DF, et al. Nivolumab plus ipilimumab versus sunitinib in advanced renal-cell carcinoma. N Engl J Med 2018;378:1277-90.

[16] Motzer RJ, Penkov K, Haanen J, et al. Avelumab plus axitinib versus sunitinib for advanced renal-cell carcinoma. $N$ Engl J Med 2019;380:1103-15

[17] Mekhail TM, Abou-Jawde RM, Boumerhi G, et al. Validation and extension of the Memorial Sloan-Kettering prognostic factors model for survival in patients with previously untreated metastatic renal cell carcinoma. J Clin Oncol 2005;23:832-41.

[18] Négrier S, Escudier B, Gomez F, et al. Prognostic factors of survival and rapid progression in 782 patients with metastatic renal carcinomas treated by cytokines: a report from the Groupe Français d'Immunothérapie. Ann Oncol 2002;13:1460-8.

[19] Negrier S, Gomez F, Douillard JY, et al. Prognostic factors of response or failure of treatment in patients with metastatic renal carcinomas treated by cytokines: a report from the Groupe Français d'Immunothérapie. World J Urol 2005;23:161-5.

[20] Quinn DI, Lara Jr PN. Renal-cell cancer-targeting an immune checkpoint or multiple kinases. N Engl J Med 2015;373:1872-4.

[21] Bersanelli M, Buti S. Cabozantinib in metastatic renal cell carcinoma: latest findings and clinical potential. Ther Adv Med Oncol 2017;9:627-36.

[22] de Peralta-Venturina M, Moch H, Amin M, et al. Sarcomatoid differentiation in renal cell carcinoma: a study of 101 cases. Am J Surg Pathol 2002;25:275-84.

[23] Amin MB, Amin MB, Tamboli P, et al. Prognostic impact of histologic subtyping of adult renal epithelial neoplasms: an experience of 405 cases. Am J Surg Pathol 2002;26:281-91.

[24] Pagliaro LC, Tannir N, Sircar K, Jonasch E. Systemic therapy for sarcomatoid renal cell carcinoma. Expert Rev Anticancer Ther 2011;11:913-20.

[25] Pécuchet N, Vieira T, Rabbe N, et al. Molecular classification of pulmonary sarcomatoid carcinomas suggests new therapeutic opportunities. Ann Oncol 2017;28:1597-604. 